\title{
Prostate Cancer Disease Characteristics at the Time of Diagnosis and Initial Treatment Offered in a Tertiary Hospital at Ouagadougou (Burkina Faso)
}

\author{
Fasnéwindé Aristide Kaboré ${ }^{1,2 *}$, Barnabé Zango ${ }^{1}$, Timothé Kambou ${ }^{2}$, Aimé Sosthène Ouédraogo ${ }^{3}$, \\ Aboubacar Bambara ${ }^{4}$, Clotaire Yaméogo ${ }^{1}$, Brahima Kirakoya ${ }^{1}$, Olga Lompo ${ }^{2,3}$ \\ ${ }^{1}$ Division of Urology, Yalgado Ouédraogo University Teaching Hospital of Ouagadougou, \\ Ouagadougou, Burkina Faso \\ ${ }^{2}$ University of Ouagadougou, Ouagadougou, Burkina Faso \\ ${ }^{3}$ Department of Pathology, Yalgado Ouédraogo University Teaching Hospital of Ouagadougou, \\ Ouagadougou, Burkina Faso \\ ${ }^{4}$ Division of Clinical Oncology, Yalgado Ouédraogo University Teaching Hospital of Ouagadougou, \\ Ouagadougou, Burkina Faso \\ Email: "kaborefamd@icloud.com
}

Received November 15, 2013; revised December 15, 2013; accepted January 3, 2014

Copyright (C 2014 Fasnéwindé Aristide Kaboré et al. This is an open access article distributed under the Creative Commons Attribution License, which permits unrestricted use, distribution, and reproduction in any medium, provided the original work is properly cited. In accordance of the Creative Commons Attribution License all Copyrights (C) 2014 are reserved for SCIRP and the owner of the intellectual property Fasnéwindé Aristide Kaboré et al. All Copyright @ 2014 are guarded by law and by SCIRP as a guardian.

\section{ABSTRACT}

Objectives: to describe the characteristics of newly diagnosed prostate cancer (PCa) and the initial treatments offered to patients in the most important urological center of Burkina Faso. Methods: We analyzed the data of a cohort of 168 consecutive patients with no prior history of PCa between January 2009 and December 2012. Diagnosis of PCa was based on histological analysis of transrectal prostate biopsies. Patient and disease characteristics and the initial treatment offered were taken in account. Results: The mean age at presentation was $68.59 \pm$ 9.41 years (range 30 to 95 years). There was a 3.6-fold increase in the incidence of PCa through the four years of the study. The mean duration of symptoms prior to presentation was $11.6 \pm 10.9$ months. The majority of cases (86.9\%) were presented as locally advanced or metastatic disease. Androgen deprivation therapy (ADT) was the first therapeutic option for 121 patients $(72 \%)$ and 73 patients $(43.4 \%)$ underwent ADT through bilateral orchiectomy. Only 3 patients $(\mathbf{1 . 7 8 \% )}$ underwent radical prostatectomy. Conclusion: An increase in the diagnosis of PCa in our country was observed in this study. The diagnosis of prostate cancer was usually tardive in Burkina Faso. Treatment often involves surgical ADT for socioeconomic reasons.

\section{KEYWORDS}

Prostrate Cancer; PSA; Presenting Symptoms; Treatment; Androgen Deprivation Therapy; Burkina Faso

\section{Introduction}

Prostate cancer (PCa) is the second most common cause of cancer and the sixth leading cause of cancer death among men worldwide with an estimated 899,000 new cases and 258,000 new deaths in 2008 [1]. The International Agency for Research on Cancer (IARC) estimates that PCa is a growing problem in Africa: approximately 57.048 African men will die of PCa in the next two decades [1,2]. Despite this dramatically increase of the dis-

\footnotetext{
${ }^{*}$ Corresponding author.
}

ease impact, PCa cases remain under diagnosed and underreported in Sub-Saharan Africa (SSA). Very few data are available for PCa incidence, prevalence, aggressiveness, and mortality in men of Sub-Saharan African countries [1-3]. In Burkina Faso the true burden of PCa is not well evaluated. Previous studies in Burkina Faso are suggestive that PCa knowledge and awareness are pour in the general population [4] and that most cases are diagnosed late [5]. The aim of the present study is to provide more data concerning characteristics of newly diagnosed PCa and an overview of initial treatments offered in the 
most important urological center of Burkina Faso.

\section{Patients and Methods}

We performed a prospective and descriptive non-randomized patient's cohort study. Newly diagnosed PCa cases were included between January 2009 and December 2012 at the urology division of the Yalgado Ouedraogo University Teaching Hospital of Ouagadougou. 168 consecutives cases were enrolled during the study period and their details entered into a database. This study did not need an ethical committee advice in Burkina Faso because it was a medical record chart abstraction study using anonymized data. All the patients had given their verbal informed consent before inclusion in the study. PCa diagnosis was done at histological analysis of transrectal prostate biopsy (TRPB) for all the patients. The variables of interest included age at diagnosis, the clinical symptoms at the time of diagnosis, serum Prostate Specific Antigen (PSA) level, the pathological variables (the histological type and the Gleason score), the stage of tumor and the initial therapeutic options. The existence of metastatic PCa was evaluated through clinical exam and thoraco-abdominal and pelvis computed tomography. Tumor stage was determined according to the 2009 TNM classification.

Descriptive statistics were used to summarize these variables in terms of frequencies, means and standard deviation. A low standard deviation (SD) indicates that the data points are close to the mean (the expected value). When the standard deviation is high, it indicates that the data points are spread out over a large range of values.

We also presented a scatter showing how the number of PCa has evolved over time, precisely from 2009 to 2012. A linear trend is estimated on this graph, showing the relationship between the time (X) and the number of PCa (Y). For this estimation, the R-Squared (R2) is calculated, showing how close the data are to the fitted regression line. The closer it is to 1 , the better the fit.

\section{Results}

\subsection{Clinical Findings}

At the time of diagnosis, the age of patients ranges from 30 to 95 years, with an average of 68,59 years and a SD of 9.41 . The majority of the patients $(76.2 \%)$ were in the 60 - 69 years and 70 - 79 years age brackets (Figure 1). 19 new cases of PCa were recorded in 2009 and the number of diagnosed cases had risen to 68 in 2012 the R2 value was 0.98033 very closer to 1 This represents a 3.6fold increase in the incidence of PCa in our institution during the four years of the study period (Figure 2). Most patients were symptomatic at the time of diagnosis (95.8\%, $n=161)$. Only 7 patients $(4.2 \%)$ were asymptomatic. Urinary retention was the first clinical patterns of reference in our center $(n=63,37.5 \%)$. Table 1 lists the

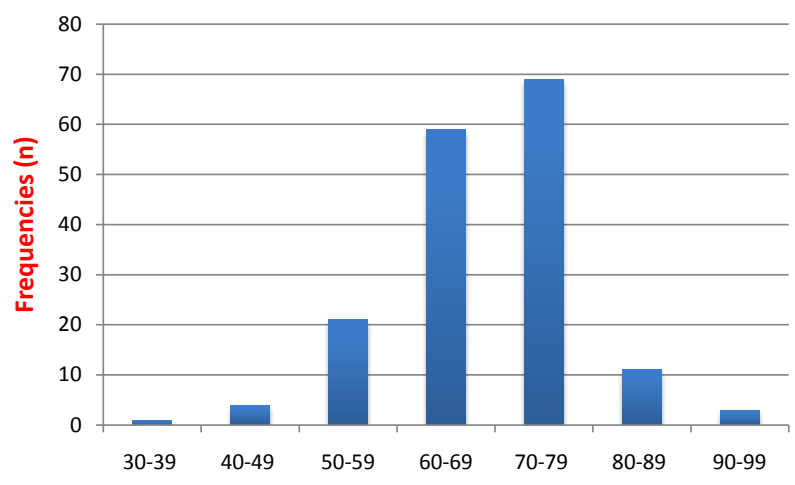

Figure 1. Age ranges of the patients.

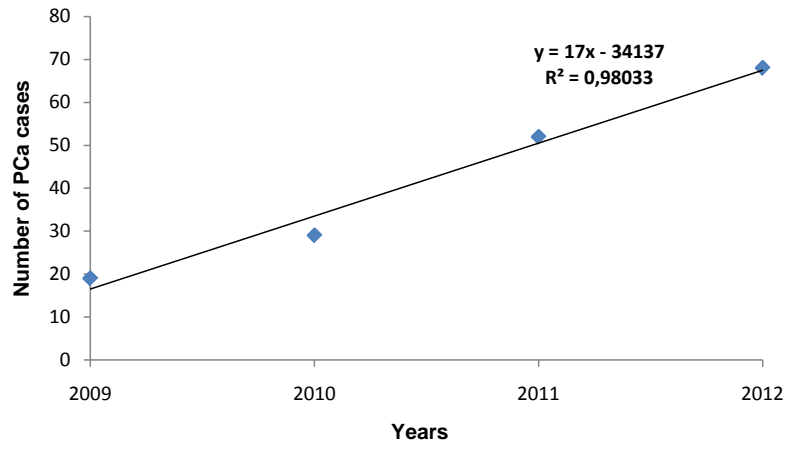

Figure 2. Evolution of the number of PCa diagnosed per year.

Table 1. Presenting symptoms at diagnosis.

\begin{tabular}{ccc}
\hline Clinical symptoms & Frequencies (n) & Percentage (\%) \\
\hline LUTS $^{*}$ urinary retention & 63 & $37.5 \%$ \\
Frequency & 60 & $35.7 \%$ \\
Dysuria & 58 & $34.5 \%$ \\
No symptoms & 7 & $4.2 \%$ \\
Bone Pain & 38 & $22.6 \%$ \\
Hematuria & 21 & $12.5 \%$ \\
\hline
\end{tabular}

*LUTS: Lower Urinary Tract Symptoms.

presenting symptoms at the time of diagnosis. The mean duration of symptoms prior to presentation was $11.6 \pm$ 10.9 months with a range of 1 week to 6 years.

The diagnosis of PCa was suspected based on increased PSA without symptoms in only 7 patients (4.2\%) and the majority of patients $(55.3 \%, \mathrm{n}=93)$ on abnormal digital rectal examination (DRE) [Table 2]. Overall mean serum PSA value was 483.3 with a standard deviation of $145.4 \mathrm{ng} / \mathrm{ml}$. The values range from 1 to 7421 and 63 patients (37.5\%) had a serum PSA level over than 100 ng/ml (Figure 3).

\subsection{PCa Diagnosis and Staging}

All the patients had adenocarcinoma on histological 


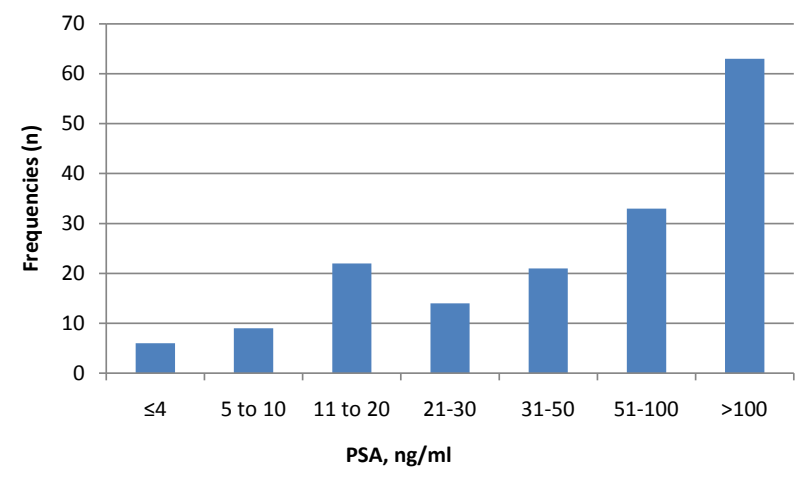

Figure 3. Repartition of patients according to serum PSA level.

Table 2. Indication of prostate biopsy.

\begin{tabular}{ccc}
\hline Increased PSA + LUTS & 68 & $40.5 \%$ \\
Abnormale DRE & 15 & $8.9 \%$ \\
Increased PSA + Abnormale DRE & 78 & $46.4 \%$ \\
Isolate increased PSA & 7 & $4.2 \%$ \\
\hline
\end{tabular}

analysis of TRPB cores (Figure 4). Most of the patients $(60.1 \%, \mathrm{n}=101)$ had Gleason score 6 and $67(39.9 \%)$ had Gleason score 7 or higher (Figure 5). Most patients $(86.9 \%, \mathrm{n}=146)$ had their primary tumor extended over the prostatic capsule (T3-T4 stages) and $13.1 \%(\mathrm{n}=22)$ had a localized disease. Table 3 summarized the tumor staging according to the 2009 TNM classification.

\subsection{Initial Treatment Offered}

Androgen deprivation therapy (ADT) was the first therapeutic option for 121 patients (72\%). A total of 73 patients (43.4\%) underwent ADT through bilateral orchiectomy that was done as monotherapy and in association with antiandrogen in 16 patients (9.5\%). 32 patients (19\%) received a medical ADT that consisted of Antiandrogen (ciproterone acetate) 200 - $300 \mathrm{mg}$ daily in 18 patients $(10.7 \%)$ and luteinizing hormone-releasing hormone (LHRH) analogue (3.75 mg monthly) in 14 (8.3\%). 14 patients (8.3\%) refused the treatment and discharged against medical advice. Only 3 patients (1.78\%) underwent radical prostatectomy. No patient had a radiation therapy because this technic was not available in Burkina Faso. The Figure 6 resumes the initials therapeutic options offered.

\section{Discussion}

Incidence of PCa varies widely between ethnic populations and countries [1]. The highest rates of this disease are in North America especially in African Americans (228.5 per 100,000 men) and Caribbean men of African descent $[1,6]$. The reported incidence rates in SSA are

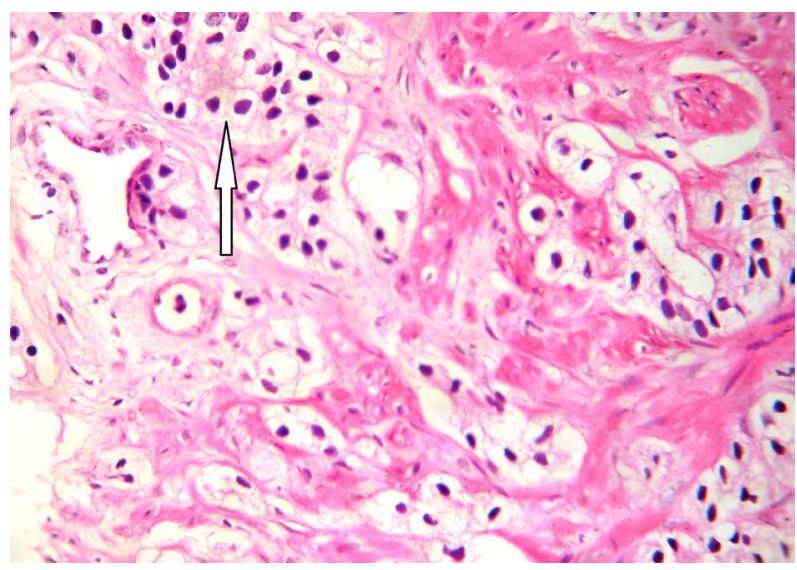

Figure 4. Microphotography $\times 100$ : Prostatic adenocarcinoma. Variation of cells sizes, enlarged and hyperchromatic nuclei (arrow). Gleason grade 3.

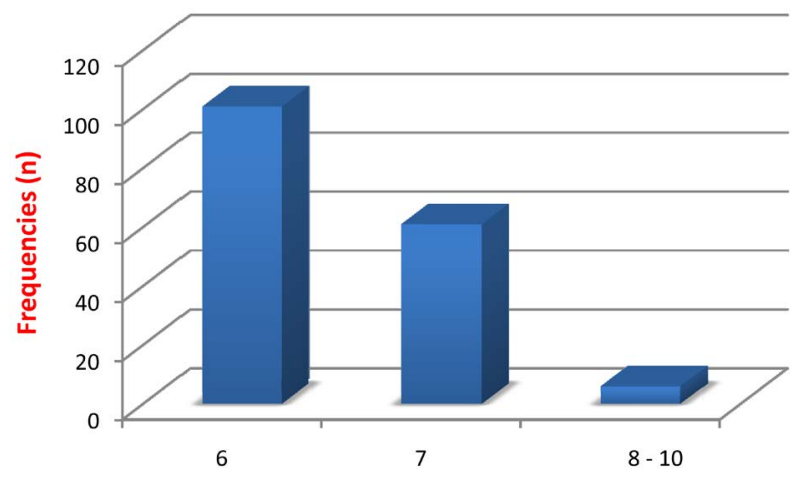

Figure 5. Repartition of patients according to Gleason score.
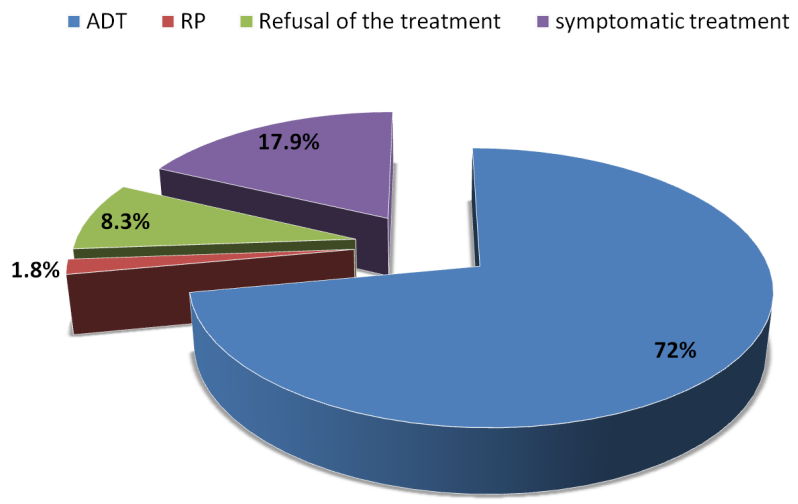

Figure 6. Initial treatment offered.

much lower than those among African Americans. Incidence varied substantially by regions in Africa with rates highest in the East (10.7 - 38.1/100,000 men-years), intermediate in the South (14.3 - 21.8), and lowest in the West $(4.7$ - 19.8) [3]. The access to quality health care services, availability of pathology services and the availability of cancer registries affect how rates of PCa are reported and can explain the differences between 
Table 3. Clinical stages according to TNM classification.

\begin{tabular}{ccc}
\hline TNM & Frequencies (n) & Percentage (\%) \\
\hline T1cN0M0 & 5 & 2.9 \\
T2aN0M0 & 2 & $1.2 \%$ \\
T2bN0M0 & 5 & $3 \%$ \\
T2cN0M0 & 4 & $2.4 \%$ \\
T2cN1M0 & 6 & $3.6 \%$ \\
T3aN0M0 & 12 & $7.1 \%$ \\
T3bN0M0 & 11 & $6.5 \%$ \\
T3bN1M1 & 20 & $12 \%$ \\
T3bN1M0 & 32 & $19 \%$ \\
T4N0M0 & 5 & $3 \%$ \\
T4N1M0 & 6 & $3.6 \%$ \\
T4N1M1 & 56 & $33.3 \%$ \\
T4N0M1 & 4 & $2.4 \%$ \\
TOTAL & $\mathbf{1 6 8}$ & $\mathbf{1 0 0 \%}$ \\
\hline
\end{tabular}

African Americans and their ancestry from SSA but also between Sub-Saharan African countries. According to those reasons we can postulate that the true PCa incidence in African men is probably higher than what is reported. This is strengthening by the global increased number of PCa cases in Africa through the last 2 decades $[1,3,7,8]$. Our study corroborates this trend with the increasing number of cases through the four years of the study (19 in 2009 to 68 in 2012).

In this study the mean age at diagnosis was $68.59 \pm$ 9.41 and patients aged 60 - 69 years and 70 - 79 years had the highest rate of PCa. The age range at the time of diagnosis of PCa in our study is also commonly reported from West Africa where studies from Ghana [9], Nigeria [10] and Senegal [11] recorded a mean age of 65.4, 66.6 and 65 years respectively. The age of diagnosis of PCa in our study is also similar to those reported from the developed world particularly in Europe and North America $[12,13]$. However, the majority of PCa cases are diagnosed in patients who are asymptomatic in the developed world due to the widespread use of the Prostate Specific Antigen (PSA) blood test [12-14]. 68.7\% of PCa cases were diagnosed in the absence of symptoms in the study of Muller [12] from the USA. Contrariwise the majority of patients in our study are symptomatic (95.8\%) and presented with LUTS and features of locally advanced or metastatic disease. Numerous studies have found that black men have a higher stage of disease at presentation and are more likely to die of their prostate cancer than their white counterparts [15-17]. Powell [17] evidenced that African Americans have similar pathogenic mechanisms and age at PCa initiation to Caucasians but they have a faster growth rate and/or an earlier transformation to clinically significant PCa. Late presentation of PCa is commonly reported from SSA [5,18-20]. The reason for the late presentation in our environment may be explained by poor awareness of PCa, poverty, inadequate diagnostic facilities and the lack of access to healthcare [2,10,11,19].

Another salient finding in the present study is the high level of serum PSA $(483.3 \pm 145.4 \mathrm{ng} / \mathrm{ml})$ at the time of diagnosis of PCa. Several studies have observed a higher age-specific serum PSA values in black men without prostate cancer compared to white men [21-23]. Despite those suggestive data, the race-specific PSA is controversial and more detailed studies are needed to elucidate if this is a result of genetic predisposition or timing in the diagnosis of PCa [21-23]. In another hand, reported prostate cancer cases from Africa shared a common elevated PSA level [10,19,24]. Niang [11] in Senegal and Heyns [24] in South Africa reported a mean PSA value of $1447.5 \mathrm{ng} / \mathrm{mL}$ and $766.1 \mathrm{ng} / \mathrm{mL}$ respectively. Except the possible race-specific PSA this could be relevant to late presentation of PCa and the absence of appropriate approaches to screening in Africa.

In a recent review on PCa incidence, aggressiveness, and mortality in men of African descent Reebeck et al. [2] found that the tumor stage distribution differed substantially by geography, with a larger proportion of stage T3/T4 tumors in Africa than other locations. Our study where stage $\mathrm{T} 3 / \mathrm{T} 4$ was predominant $(86.9 \%)$ is coherent with the findings of this review. Advanced and highgrade PCa among black men from the USA, Caribbean and other sub-Saharan African countries suggests an element of shared genetics disadvantage of high-risk disease but may also be due to differences in screening and availability of diagnosis resources(PSA, pathologists, urologists) $[2,15,25]$.

Bilateral orchiectomy is a common and widely used surgical procedure for advanced and metastatic PCa in Burkina Faso and generally in the developing world. At the opposite, the use of surgical ADT is declining in developed countries due to the availability of medical ADT which are considered to be equally effective in reducing testosterone levels as orchiectomy with less psychological effect [26,27]. Surgical orchidectomy is a less expensive treatment comparatively with medical ADT. This is the mains reason that justifies the widespread use of bilateral orchidectomy in SSA [11,19,23]. Moreover many patients in our environment are reluctant to receive medication $(8.3 \%$ of patients in our study refused the treatment) and lost to follow-up after hospitalization. Therefore, bilateral orchiectomy is a single and safe method to ensure that treatment is maintained. 
Radical prostatectomy remains the gold standard for treatment of organ confined prostate cancer [28,29]. Only 3 radical prostatectomies (1.8\% of patients) were done in our study. It has been noted that access of patients with localized PCa to radical prostatectomy in SSA is low mainly due to lack of expertise in the procedure of open radical prostatectomy and advanced disease at diagnosis $[11,23,27]$. However curative management of prostate cancer through radical prostatectomy and radiotherapy depends on early detection of the disease. The development of a well-planned national screening program can be a way to increase early diagnosis and improve PCa outcomes in Burkina Faso and other countries in SSA.

The mains limitations of this study are: 1 ) data reported here are limited to one medical center and are not representative of all the population of Burkina Faso; 2) PSA testing and prostate biopsy are not readily available and many PCa are probably excluded in this study. Despite those limitations this study have the merit to complete data from Burkina Faso and provide an overview of newly diagnosed PCa characteristics and treatment in the most important referral center for patients with urological diseases from all over the country. Further multicentric studies are needed to better access the PCa epidemiology disease characteristics and treatment outcomes in Burkina Faso.

\section{Conclusion}

An increase in the diagnosis of PCa in our country was observed in this study. The diagnosis of prostate cancer was usually tardive in Burkina Faso. Treatment often involves surgical ADT for socioeconomic reasons. Prostatectomy was only very seldom indicated. Screening for PCa could help detect earlier stage PCa and improve the treatment outcome in Burkina Faso.

\section{REFERENCES}

[1] J. Ferlay, H. R. Shin, F. Bray, D. Forman, C. Mathers and D. M. Parkin, "Estimates of Worldwide Burden of Cancer in 2008: GLOBOCAN 2008,” International Journal of Cancer, Vol. 127, No. 12, 2010, pp. 2893-2917. http://dx.doi.org/10.1002/ijc.25516

[2] T. R. Rebbeck, S. S. Devesa, B.-L. Chang, et al., “Global Patterns of Prostate Cancer Incidence, Aggressiveness, and Mortality in Men of African Descent," Prostate Cancer, Vol. 2013, 2013, Article ID: 560857. http://dx.doi.org/10.1155/2013/560857

[3] L. W. Chu, J. Ritchey, S. S. Devesa, S. M. Quraishi, H. Zhang and A. W. Hsing, "Prostate Cancer Incidence Rates in Africa,” Prostate Cancer, Vol. 2011, 2011, Article ID: 947870.

[4] F. A. Kabore, T. Kambou, B. Zango and A. Ouédraogo, "Knowledge and Awareness of Prostate Cancer among the General Public in Burkina Faso," Journal of Cancer
Education, 2013, in press. http://dx.doi.org/10.1007/s13187-013-0545-2

[5] F. A. Kabore, B. Zango, A. Sanou, C. Yameogo and B. Kirakoya, "Prostate Cancer Outcome in Burkina Faso," Infectious Agents and Cancer, Vol. 6, Suppl. 2, 2011, p. S6. http://dx.doi.org/10.1186/1750-9378-6-S2-S6

[6] SEER Cancer Statistics Factsheets, "Prostate Cancer," National Cancer Institute, Bethesda. http://seer.cancer.gov/statfacts/html/prost.html

[7] E. Chokunonga, M. Z. Borok, Z. M. Chirenje, A. M. Nyakabau and D. M. Parkin, "Trends in the Incidence of Cancer in the Black Population of Harare, Zimbabwe 19912010,” International Journal of Cancer, Vol. 133, No. 3, 2013, pp. 721-729. http://dx.doi.org/10.1002/ijc.28063

[8] G. O. Ifere, F. Abebe and G. A. Ananaba, "Emergent Trends in the Reported Incidence of Prostate Cancer in Nigeria," Clinical Epidemiology, Vol. 4, No. 1, 2012, pp. 19-32. http://dx.doi.org/10.2147/CLEP.S23536

[9] J. Yarney, V. Vanderpuye and J. Mensah, "Clinicopathologic Features and Determinants of Gleason Score of Prostate Cancer in Ghanaian Men," Urologic Oncology: Seminars and Original Investigations, Vol. 31, No. 3, 2013, pp. 325-330. http://dx.doi.org/10.1016/j.urolonc.2011.01.018

[10] A. A. Ajape, K. O. Ibrahim, J. A. Fakeye and O. O. Abiola, “An Overview of Cancer of the Prostate Diagnosis and Management in Nigeria: The Experience in a Nigerian Tertiary Hospital,” Annals of African Medicine, Vol. 9, No. 3, 2010, pp. 113-117. http://dx.doi.org/10.4103/1596-3519.68353

[11] L. Niang, M. Ndoye, A. Ouattara, M. Jalloh, M. Labou, I. Thiam, S. C. Kouka, J. J. Diaw and S. M. Gueye, "Management of Prostate Cancer in Senegal: What Is Being Done?” Progrès en Urologie, Vol. 23, No. 1, 2013, pp. 36-41. http://dx.doi.org/10.1016/j.purol.2012.09.002

[12] D. C. Miller, M. S. Litwin, J. Bergman, et al., "Prostate Cancer Severity among Low Income, Uninsured Men," The Journal of Urology, Vol. 181, No. 2, 2009, pp. 579584. http://dx.doi.org/10.1016/j.juro.2008.10.010

[13] D. C. Greenberg, K. A. Wright, A. Lophathanon, K. R. Muir and V. J. Gnanapragasam, "Changing Presentation of Prostate Cancer in a UK Population-10 Years Trends in Prostate Cancer Risk Profiles in the East of England," British Journal of Cancer, Vol. 109, 2013, pp. 2115-2120. http://dx.doi.org/10.1038/bjc.2013.589

[14] M. J. Roobol and F. H. Schröder, "European Randomized Study of Screening for Prostate Cancer: Achievements and Presentation,” BJU International, Vol. 92, Suppl. S2, 2003, pp. 117-122. http://dx.doi.org/10.1111/j.1464-410X.2003.4698x.x

[15] S. Punnen and M. R. Cooperberg, "The Epidemiology of High-Risk Prostate Cancer,” Current Opinion in Urology, Vol. 23, No. 4, 2013, pp. 331-336. http://dx.doi.org/10.1097/MOU.0b013e328361d48e

[16] B. A. Virnig, N. N. Baxter, E. B. Habermann, et al., “A Matter of race: Early- versus Late-Stage Cancer Diagnosis," Health Affairs (Millwood), Vol. 28, No. 1, 2009, pp. 160-168. http://dx.doi.org/10.1377/hlthaff.28.1.160 
[17] I. J. Powell, C. H. Bock, J. J. Ruterbusch, et al., "Evidence Supports a Faster Growth Rate and/or Earlier Transformation to Clinically Significant Prostate Cancer in Black Than in White American Men, and Influences Racial Progression and Mortality Disparity,” The Journal of Urology, Vol. 183, No. 5, 2010, pp. 1792-1796. http://dx.doi.org/10.1016/j.juro.2010.01.015

[18] M. K. Sapira and C. C. Obiorah, "Age and Pathology of Prostate Cancer in South-Southern Nigeria; Is There a Pattern?” Medical Journal of Malaysia, Vol. 67, No. 4, 2012, pp. 417-419.

[19] S. M. Gueye, C. M. Ziegler-Johnson, T. Friebel, et al., "Clinical Characteristics of Prostate Cancer in African Americans, American Whites, and Senegalese Men," Urology, Vol. 61, No. 5, 2003, pp. 987-992. http://dx.doi.org/10.1016/S0090-4295(02)02588-8

[20] K. Yamoah, K. Beecham, S. E. Hegarty, T. Hyslop, T. Showalter and J. Yarney, "Early Results of Prostate Cancer Radiation Therapy: An Analysis with Emphasis on Research Strategies to Improve Treatment Delivery and Outcomes,” BMC Cancer, Vol. 13, 2013, p. 23. http://dx.doi.org/10.1186/1471-2407-13-23

[21] K. A. Cooney, M. S. Strawderman, K. J. Wojno, K. M. Doerr, A. Taylor, K. H. Alcser, S. G. Heeringa, J. M. Taylor, J. T. Wei, J. E. Montie and D. Schottenfeld, "Age-Specific Distribution of Serum Prostate-Specific Antigen in a Community-Based Study of African-American Men,” Urology, Vol. 57, No. 1, 2001, pp. 91-96. http://dx.doi.org/10.1016/S0090-4295(00)00873-6

[22] D. M. Preston, L. I. Levin, D. J. Jacobson, et al., "Prostate-Specific Antigen Levels in Young White and Black Men 20 to 45 Years Old,” Urology, Vol. 56, No. 5, 2000, pp. 812-816. http://dx.doi.org/10.1016/S0090-4295(00)00764-0

[23] S. Sutcliffe, R. Pakpahan, L. J. Sokoll, D. J. Elliott, R. L. Nevin, S. B. Cersovsky, P. C. Walsh and E. A. Platz, "Prostate-Specific Antigen Concentration in Young Men:
New Estimates and Review of the Literature," BJU International, Vol. 110, No. 11, 2012, pp. 1627-1635. http://dx.doi.org/10.1111/j.1464-410X.2012.11111.x

[24] C. F. Heyns, M. Fisher, A. Lecuona and A. van der Merwe, "Prostate Cancer among Different Racial Groups in the Western Cape: Presenting Features and Management,” South African Medical Journal, Vol. 101, 2011, pp. 267-270.

[25] S. A. Fedewa and A. Jemal, "Prostate Cancer Disease Severity and Country of Origin among Black Men in the United States,” Prostate Cancer and Prostatic Disease, Vol. 16, 2013, pp. 176-180. http://dx.doi.org/10.1038/pcan.2012.53

[26] M. Grossmann, A. S. Cheung and J. D. Zajac, “Androgens and Prostate Cancer; Pathogenesis and Deprivation Therapy,” Best Practice \& Research Clinical Endocrinology \& Metabolism, Vol. 27, No. 4, 2013, pp. 603-616. http://dx.doi.org/10.1016/j.beem.2013.05.001

[27] C. J. DiBlasio, J. B. Malcolm, J. Hammett, J. Y. Wan, M. A. Aleman, A. L. Patterson, R. W. Wake and I. H. Derweesh, "Survival Outcomes in Men Receiving Androgendeprivation Therapy as Primary or Salvage Treatment for Localized or Advanced Prostate Cancer: 20-Year Single-Centre Experience,” BJU International, Vol. 104, No. 9, 2009, pp. 1208-1214. http://dx.doi.org/10.1111/j.1464-410X.2009.08593.x

[28] M. Y. Kyei, E. J. Mensah, S. Gepi-Attee, D. Kwami, K. Ampadu, E. Asante, G. O. Klufio and E. D. Yeboah, "Outcomes after Radical Prostatectomy in Ghanaians: A Surgeon's Early Experience,” ISRN Urology, Vol. 2013, 2013, Article ID: 832496.

[29] P. C. Walsh, "Radical Prostatectomy for Localized Prostate Cancer Provides Durable Cancer Control with Excellent Quality of Life: A Structured Debate,” The Journal of Urology, Vol. 163, No. 6, 2000, pp. 1802-1807. http://dx.doi.org/10.1016/S0022-5347(05)67547-7

\author{
Abbreviations \\ PCa: Prostate Cancer \\ SSA: Sub-Saharan Africa \\ TRPB: Transrectal Prostate Biopsy \\ DRE: Digital Rectal Examination \\ PSA: ProstateSpecific Antigen \\ ADT: Androgen Deprivation Therapy
}

LUTS: Lower Urinary Tract Symptoms 